\title{
Aberrant DNA methylation at imprinted genes in testicular sperm retrieved from men with obstructive azoospermia and undergoing vasectomy reversal
}

\author{
Agata Minor, Victor Chow and Sai Ma \\ Department of Obstetrics and Gynaecology, BC Women's Hospital and Health Centre, University of British Columbia, \\ Room D414B, D6-4500 Oak Street, Vancouver, British Columbia, Canada V6H 3N1 \\ Correspondence should be addressed to S Ma; Email: sai@interchange.ubc.ca
}

\begin{abstract}
Male factor infertility has been associated with abnormal DNA methylation at imprinted genes. Little information is available on the status of imprinting in the sperm of men with azoospermia, including the association between aberrant imprinting and obstructive azoospermia (OA) or non-OA (NOA). Analysis of DNA methylation at imprinted genes in the sperm of men undergoing vasectomy reversal would aid determination of whether aberrant imprinting is associated with obstruction. Testicular sperm was retrieved from testicular biopsies obtained from men with azoospermia $(N=18)$, including $O A(N=10)$, NOA $(N=5)$, and unknown pathology $(N=3)$, and from men undergoing vasectomy reversal $(N=17)$. Sperm was also obtained from proven fertile men $(N=9)$. DNA methylation was investigated at multiple CpG sites within the differentially methylated regions (DMRs) of three imprinted genes, H19, IG-GTL2 and MEST, using bisulphite sequencing. Unique clones representative of single cells were analyzed. We found a significant decrease in DNA methylation at the H19 DMR in testicular sperm of azoospermic men compared with proven fertile men. The decrease was also significant between $\mathrm{OA}$ and proven fertile men, and between men undergoing vasectomy reversal and proven fertile men, suggesting that aberrant DNA methylation may be associated with obstruction. Changes in DNA methylation at IG-GTL2 and MEST DMRs among groups were not significant. Our data suggest that imprinting abnormalities may be associated with obstruction and may occur in response to changes in testicular environment and not only spermatogenesis failure, as previously reported. Methylation at the H19 DMR was particularly prone to modification in testicular sperm.
\end{abstract}

Reproduction (2011) 141 749-757

\section{Introduction}

Azoospermia is defined as the absence of sperm from the ejaculate, which affects an estimated $10-20 \%$ of infertile men (Jarow et al. 1989). It results from obstruction in $40 \%$ of cases, while non-obstructive azoospermia (NOA) due to spermatogenesis failure is seen in the remaining cases (Jarow et al. 1989). Although, in these men, sperm is absent from the ejaculate, it may be retrieved from the testes and used to achieve pregnancy through ICSI. Recent reports have demonstrated the presence of epigenetic abnormalities in the sperm of infertile men (Kobayashi et al. 2007, Marques et al. 2008, Boissonnas et al. 2010), including those affected by azoospermia (Marques et al. 2010).

The epigenome refers to the histone and DNA methylation patterns established in a cell that control gene expression. DNA methylation is the covalent addition of a methyl group to cytosines located within CpG dinucleotides. About $90 \%$ of DNA methylation in the genome occurs at repetitive sequences (Yoder et al. 1997).
DNA methylation also marks the differentially methylated regions (DMRs) of imprinted genes, allowing for mono-allelic parent-specific gene expression. Proper DNA methylation is an integral part of spermatogenesis progression (Raman \& Narayan 1995, Yaman \& Grandjean 2006). Once DNA methylation at imprinted genes in the male gametes is established prior to entry into meiosis, it is maintained throughout development (Olek \& Walter 1997, Kerjean et al. 2000). Abnormal DNA methylation at imprinted genes has been associated with spermatogenesis failure, primarily in men affected by moderate oligozoospermia (Kobayashi et al. 2007, Marques et al. 2008, Boissonnas et al. 2010), while DNA methylation at repetitive sequences appears properly set (Kobayashi et al. 2007, Marques et al. 2008). Although DNA methylation at imprinted genes in testicular sperm of men with azoospermia has been previously studied, an association between aberrant imprinting and NOA or obstructive azoospermia (OA) has not been established (Marques et al. 2010). Analysis of DNA methylation at imprinted genes in the sperm 
retrieved from men with different pathologies, NOA and OA, would help in the understanding of factors that may disrupt DNA methylation such as spermatogenesis failure in NOA patients or obstruction in OA patients. Furthermore, obstruction may be either associated with the congenital bilateral absence of the vas deferens (CBADV) or induced by vasectomy in previously fertile men. No data are currently available on the status of DNA methylation at imprinted genes in the sperm of vasectomized men. Study of DNA methylation at imprinted genes in these men would also help to determine whether aberrant imprinting is associated with obstruction.

While most imprinted genes are methylated in the oocyte, including MEST (Lucifero et al. 2002), two imprinted genes, $\mathrm{H} 19$ and the intragenic region (IG) of GTL2, show sperm-specific DNA methylation (Kerjean et al. 2000, Geuns et al. 2007). The H19/IGF2, GTL2/ DLK1 regions and MEST are important regulators of prenatal growth (DeChiara et al. 1990, Kaneko-Ishino et al. 1995, Georgiades et al. 2000, 2001). Abnormal methylation within the three DMRs has been associated with imprinting syndromes observed in children (Kagami et al. 2007, Kanber et al. 2009) and abortuses achieved through the use of ICSI (Kobayashi et al. 2009). Therefore, abnormal methylation at imprinted genes in the sperm may not only be associated with male factor infertility but may also be passed on to the progeny through the use of ICSI and affect pregnancy outcome.

In this study, DNA methylation at the DMRs of three imprinted genes, H19, IG-GTL2 and MEST, was evaluated in the testicular sperm retrieved from men affected by azoospermia, NOA and OA, men undergoing vasectomy reversal, and in the ejaculate sperm from normozoospermic men of proven fertility. DNA methylation was carried out using bisulphite sequencing so that DNA methylation at multiple CpG sites could be simultaneously analyzed and DNA methylation could be visualized at the single sperm level. We hypothesized that a higher incidence of abnormal DNA methylation at imprinted genes would be identified in testicular sperm of azoospermic men and in men undergoing vasectomy reversal compared with proven fertile men. Based on the limited data available, we also hypothesized that sperm obtained from men affected by obstruction would be more prone to methylation abnormalities at imprinted genes compared with sperm retrieved from men affected by NOA.

\section{Results}

\section{Clinical information}

The vasectomy reversal men were significantly older compared with the proven fertile men $(P<0.0001)$. The age difference between proven fertile men, azoospermic men, OA men, and NOA men was not significant ( $P=0.14, P=0.16$ and $P=0.13$ respectively). We found a significant age difference between the two groups affected by obstruction: the vasectomy reversal and OA men $(P=0.0002)$.

\section{Analysis of methylation at DMRs of imprinted genes}

In total, 1167 clones were analyzed, with $77.3 \%$ being unique: $75.4 \%$ of clones in the vasectomy reversal group, $76.1 \%$ of clones in the azoospermia groups and $82.7 \%$ of clones in the proven fertile group were unique. An average of 4.5, 4.3 and 2.9 amplification reactions was performed per sample in the vasectomy reversal group, azoospermia group and the proven fertile group respectively. An average of $6.7,6.5$ and 8.0 unique clones was analyzed for each sample in the vasectomy reversal group, azoospermia group and the proven fertile group respectively. In some cases, multiple amplification reactions failed and due to a limited amount of sample available fewer clones could be analyzed. Unique clones analyzed for the samples are indicated in Fig. 1 and are shown directly in the diagram. Most of the unique clones originated from different amplification

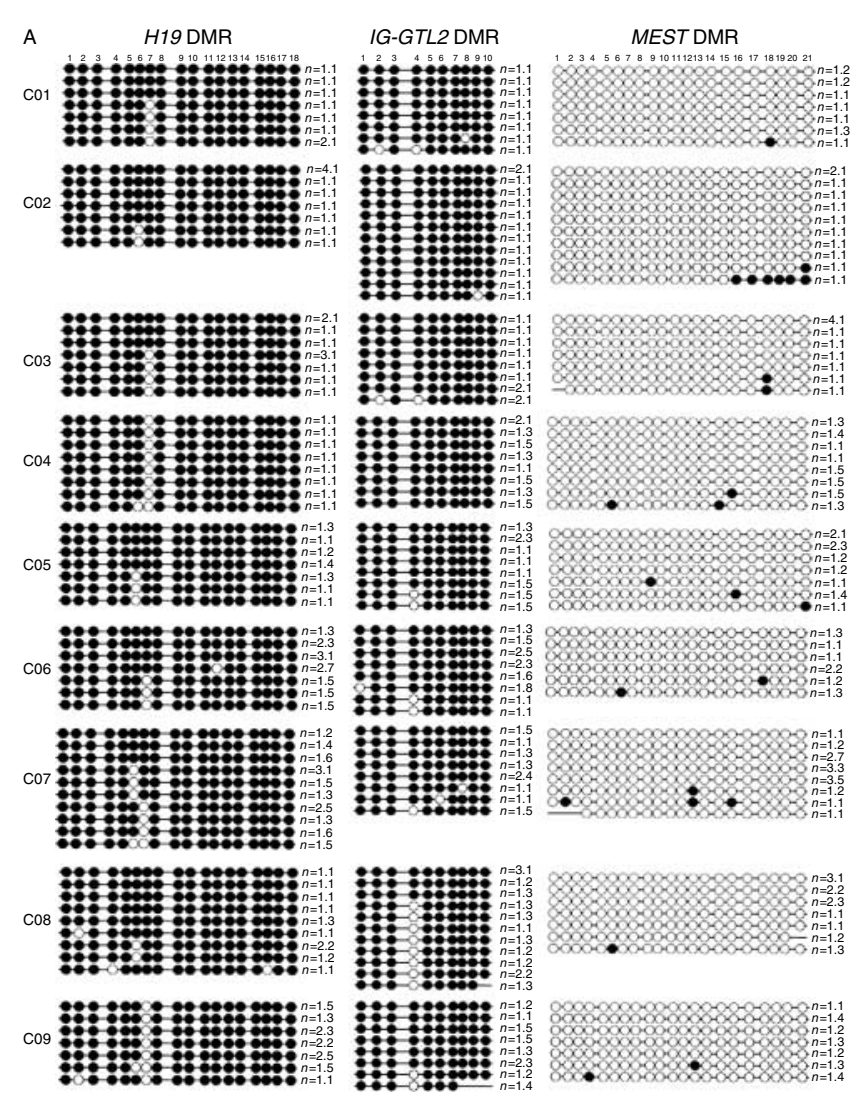

Figure 1 (legend continued) 

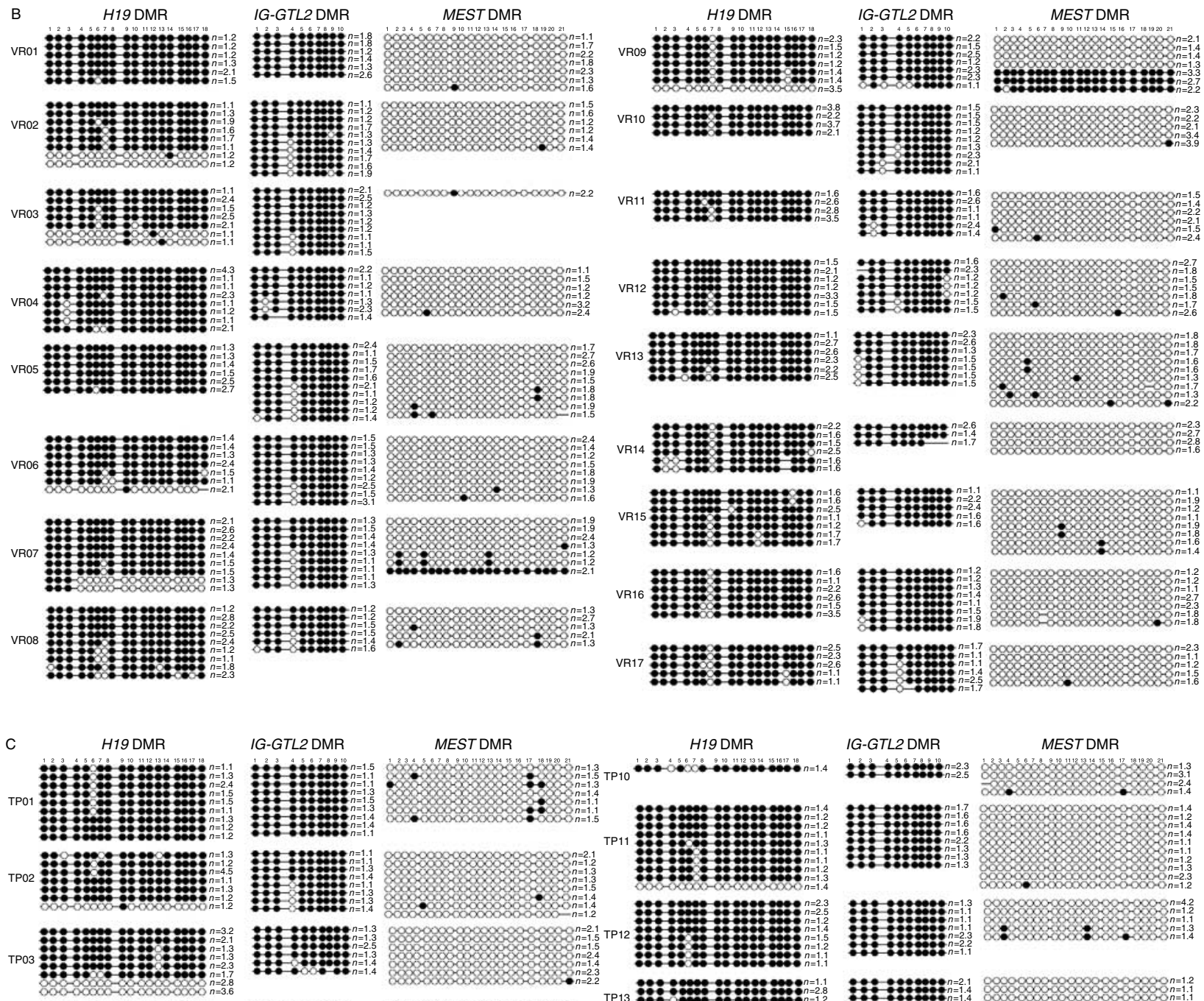

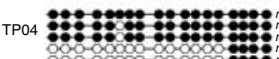
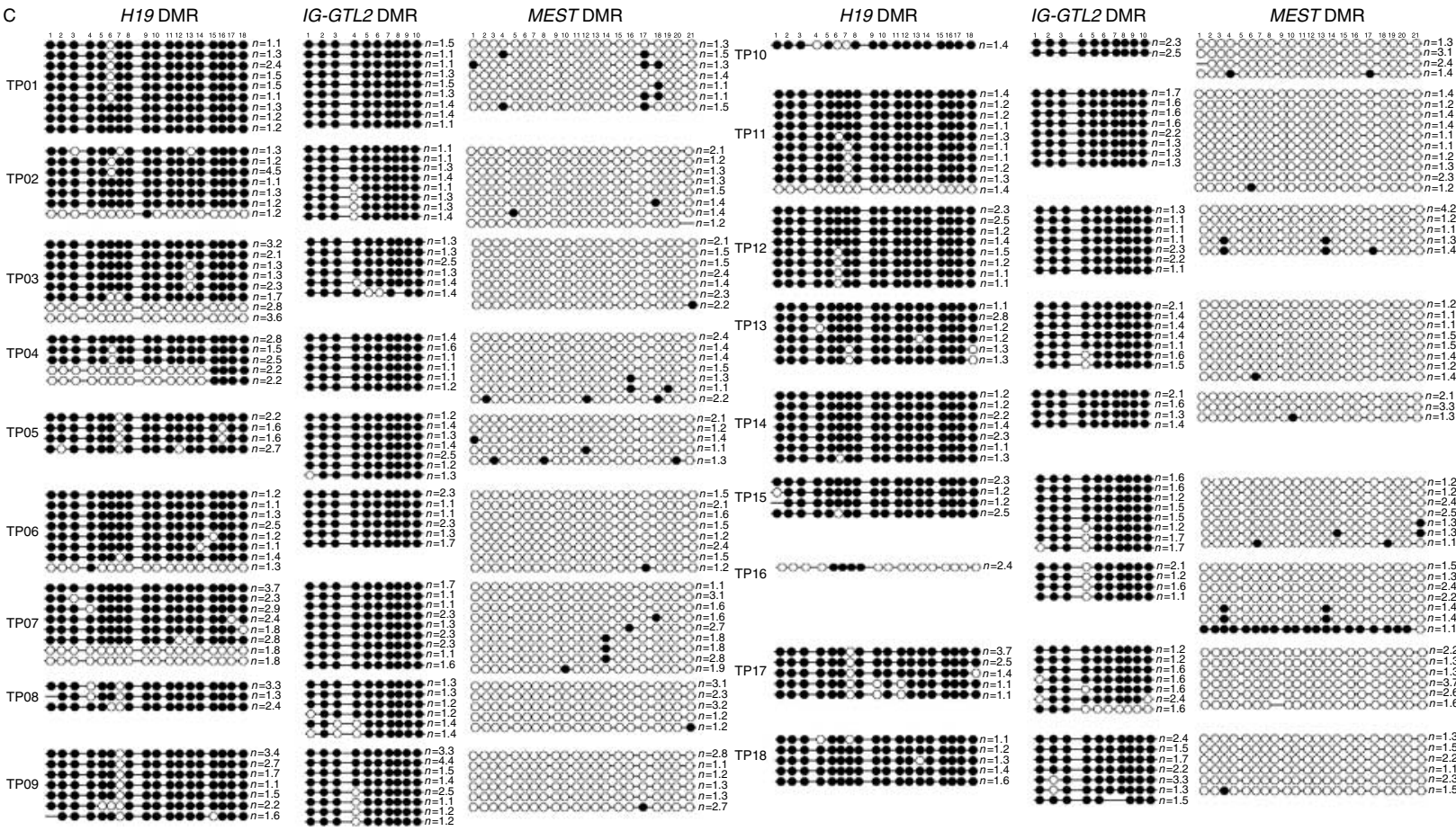

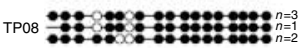
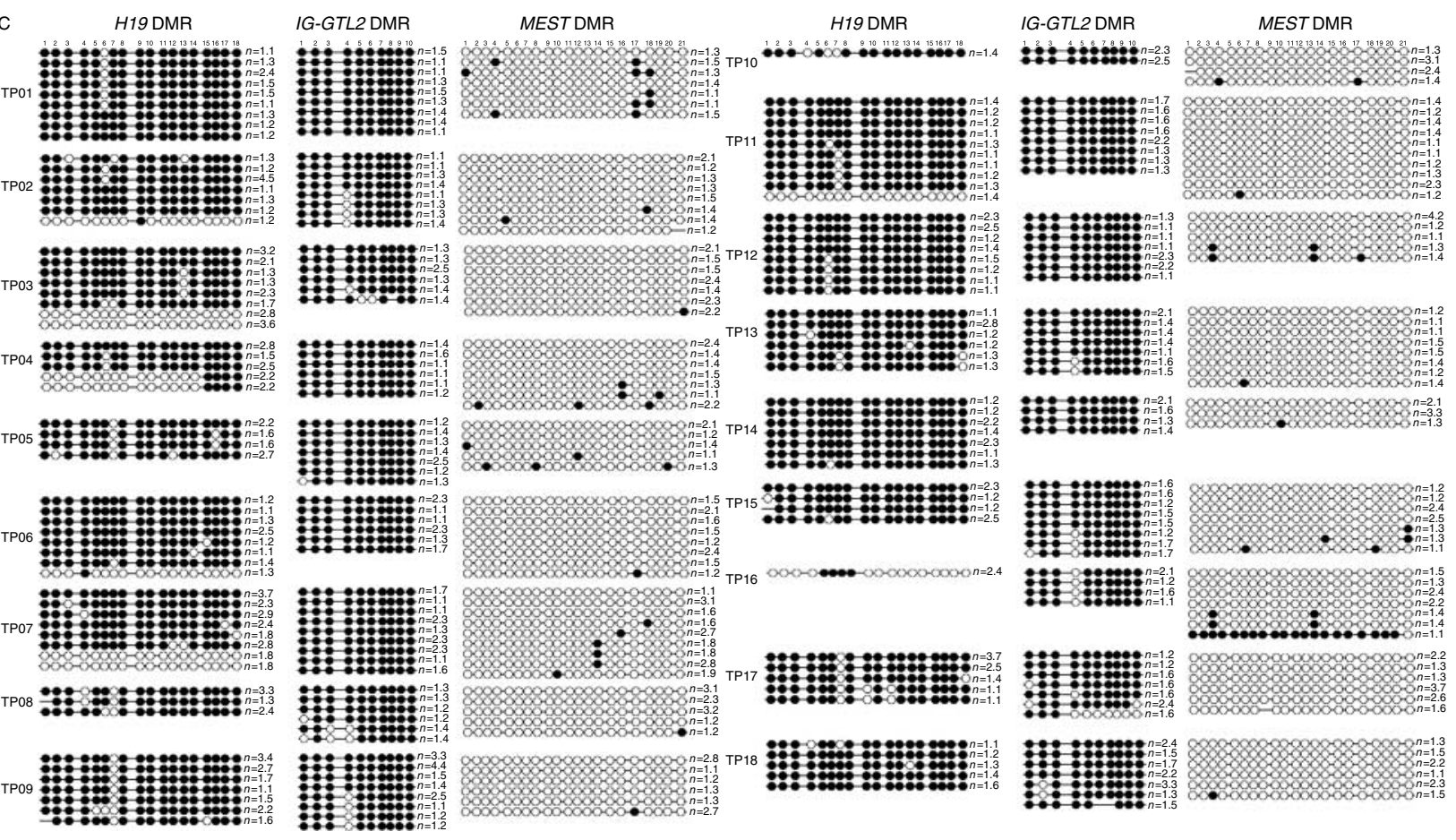

Figure 1 Bead diagrams representing methylation at CpG sites studied at H19, IG-GTL2, and MEST differentially methylated regions (DMRs). Methylated (black bead) and unmethylated (open bead) status of each CpG site is indicated within the studied sequences in (A) proven fertile men (C01-C09), (B) vasectomy reversal men (VR01-VR17), and in (C) men affected by azoospermia (TP01-TP18). Each strand shown indicates the methylation profile of a sperm. Unique clones analyzed at each DMR are shown directly in the diagram, and are coded on the right-hand side with the first number designating the number of non-unique clones that were analyzed for each sequence, followed by the amplification reaction each clone came from. As can be observed, most unique clones originated from different amplification reactions. Missing beads represent $\mathrm{CpG}$ sites that could not be analyzed. The amplification reactions are not necessarily labeled in consecutive order. 
reactions. On the basis of these results, the DNA methylation level was calculated for each sample at each DMR analyzed (Fig. 2). The absence of ejaculate or testicular sperm with improperly methylated IG-GTL2 DMR, with the exception of one sample, suggests that the sperm population was not contaminated with nongerm cells, as these also carry a non-methylated imprint at the IG-GTL2 DMR.
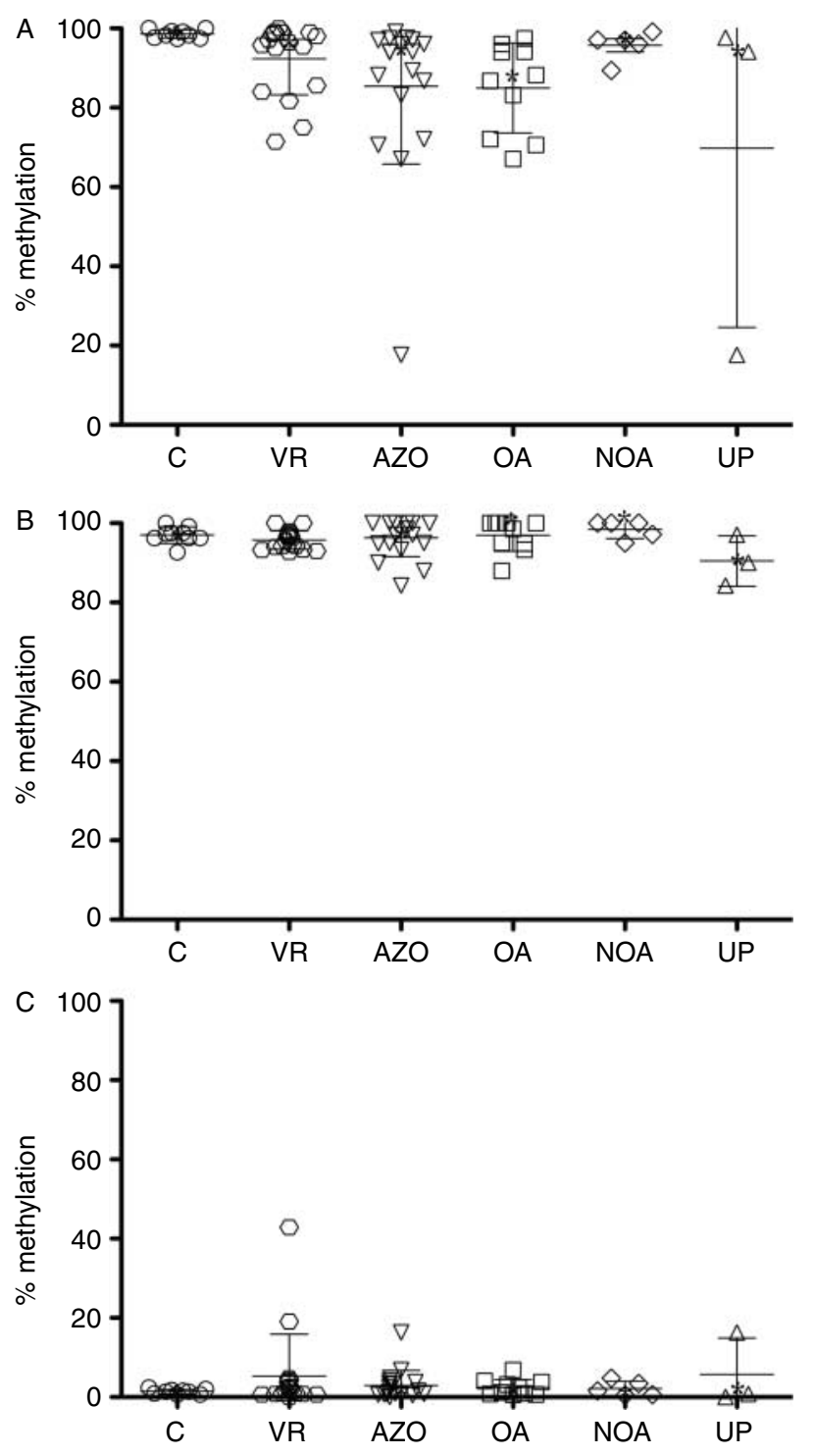

Figure 2 DNA methylation level at imprinted genes in vasectomy reversal and azoospermia groups. The methylation level (\% methylation) is shown for each sample analyzed within the (A) H19 DMR, (B) IG-GTL2 DMR, and (C) MEST DMR. Methylation level was analyzed in proven fertile men $(C)$, in men undergoing vasectomy reversal (VR), and in men affected by azoospermia (AZO). The AZO group was further subdivided into three subgroups: obstructive and non-obstructive azoospermia (OA and NOA respectively), and of unknown pathology (UP). The horizontal lines indicate the group means and the whiskers indicate S.D. of the group means. ${ }^{*}$ Indicates the median.

\section{Methylation at the H19 DMR}

In the proven fertile men, the methylation level ranged between 97.39 and $100 \%$ and hypomethylated unique clones were not detected (Figs $1 \mathrm{~A}$ and 2). The methylation level at the H19 DMR ranged between 71.4 and $100 \%$ in the vasectomy reversal group (Figs 1B and 2). Hypomethylated unique clones were found in five of the 17 vasectomy reversal samples: either one or two unique clones were hypomethylated or completely non-methylated in these samples (Fig. 1B). In the azoospermic patient group, the methylation level at the H19 DMR ranged between 17.65 and $99.16 \%$ (Fig. 2). A methylation level of $17.65 \%$ was found in a sample of unknown pathology (TP16); however, only one unique and two non-unique clones could be analyzed that may not be representative of the overall methylation level in that sample (Fig. 1C). In total, hypomethylated unique clones were found in six of the 18 azoospermic patient samples: in five of the 10 OA samples (TP02, TP03, TP04, TP06, and TP07) and in one sample of unknown pathology (TP16; Fig. 1C). The incidence of abnormal methylation at the H19 DMR was significantly higher in the azoospermia patient group compared with the proven fertile men (7/18 vs 0/9, Fisher's exact test, $P=0.036$; Table 1). The incidence was also higher in the OA group compared with the proven fertile men (5/10 vs $0 / 9$, Fisher's exact test, $P=0.022$ ) (Table 1 ). No other significant differences were found between groups at the H19 DMR.

We found a significant decrease in methylation at the H19 DMR in the vasectomy reversal group compared with the proven fertile men (Mann-Whitney (MW), $P=0.0165)$. However, the significance was lost after the post hoc Dunn's correction following ANOVA (Kruskal-Wallis (KW), $P>0.05)$. We also found a significant decrease in methylation at the H19 DMR in the azoospermic patient group compared with the proven fertile men $(\mathrm{KW}, P<0.01)$. Furthermore, there was a significant decrease in methylation at the $\mathrm{H} 19$ DMR between the OA group and the proven fertile men $(K W, P<0.01)$. The difference in the methylation level at the H19 DMR between other groups was not significant $(\mathrm{KW}, \mathrm{P}>0.05)$.

\section{Methylation at the IG-GTL2 DMR}

The methylation level at the IG-GTL2 DMR ranged between 92.8 and $100 \%, 84.29$ and 100\%, 92.66 and $100 \%$ in the vasectomy reversal group, azoospermic group and proven fertile men respectively (Fig. 2). Hypomethylation at the IG-GTL2 DMR affecting one of seven unique clones analyzed was found in one azoospermic patient sample of unknown pathology (TP17) (Fig. 1C). Hypomethylation at the IG-GTL2 DMR was not found in the vasectomy reversal group or the proven fertile group (Fig. 1). There were no 
Table 1 Aberrant imprinting in the sperm of men with azoospermia and vasectomy reversal.

\begin{tabular}{lccc}
\hline & \multicolumn{3}{c}{ DMR analyzed } \\
\cline { 2 - 4 } Study group & $H 19$ & IG-GTL2 & MEST \\
\hline Proven fertile men & $0 / 9$ & $0 / 9$ & $0 / 9$ \\
Vasectomy reversal & $5 / 17(29.4)$ & $0 / 17$ & $2 / 17(11.8)$ \\
AZO & $7 / 18(38.9)^{*}$ & $1 / 18(5.5)$ & $1 / 18(5.5)$ \\
OA & $5 / 10(50)^{*}$ & $0 / 10$ & $0 / 10$ \\
NOA & $1 / 5(20)$ & $0 / 5$ & $0 / 5$ \\
Unknown pathology & $1 / 3(33.3)$ & $1 / 3(33.3)$ & $1 / 3(33.3)$ \\
\hline
\end{tabular}

Percentages shown in brackets. *Significantly different compared with proven fertile men (Fisher's exact test, $P<0.05$ ). DMR, differentially methylated region; AZO, azoospermia; OA, obstructive azoospermia; NOA, non-obstructive azoospermia.

significant differences in the incidence of abnormal methylation at the IG-GTL2 DMR between any of the groups studied (Table 1). The difference in the methylation level at the IG-GTL2 DMR between any of the groups studied was not significant (KW, $P>0.05)$.

\section{Methylation at the MEST DMR}

The methylation level at the MEST DMR ranged between 0 and $42.9 \%, 0$ and $16.33 \%, 0.7$ and $2.4 \%$ in the vasectomy reversal group, azoospermia group and men of proven fertility respectively (Fig. 2). Hypermethylated unique clones were found in two samples from the vasectomy reversal group (VR07 and VR09), and in one sample from the azoospermic patient group in a sample with unknown pathology (TP16) (Fig. 1). Hypermethylated unique clones were not observed in any of the proven fertile group (Table 1). Abnormal methylation at the MEST DMR was found in $11.8 \%$ (2/17) of vasectomy reversal samples and in 5.5\% (1/18) of the azoospermia patient group (Table 1). There were no significant differences in the incidence of abnormal methylation at the MEST DMR between any of the groups studied. The difference in the methylation level at the MEST DMR between any of the groups studied was not significant (KW, $P>0.05)$.

Three of the 12 samples with abnormal methylation at the H19 DMR (VR07, VR09, and TP16) also had abnormal methylation at the MEST DMR. The one patient with abnormal methylation at the IG-GTL2 DMR (TP17) had normal methylation at the H19 and the MEST DMRs.

\section{Discussion}

In this study, testicular sperm were isolated from testicular tissue for analysis of DNA methylation at imprinted genes. Our results demonstrate a significant decrease in DNA methylation at the H19 DMR in men with azoospermia and in men with OA compared with men of proven fertility. We also found a significant decrease in DNA methylation at the H19 DMR in men undergoing vasectomy reversal compared with proven fertile men. The loss of methylation at the H19 DMR in the testicular sperm of men after undergoing a vasectomy reversal and in OA men suggests that the loss of methylation may be associated with obstruction. Furthermore, we observed a significant decrease in DNA methylation at the H19 DMR in previously fertile men, those undergoing vasectomy reversal, and in infertile men, those affected by OA. The difference in DNA methylation between the two groups at the H19 DMR was not significant, and suggests that DNA methylation at imprinted genes was not associated with the fertility status of the patients. Differences in DNA methylation at the IG-GTL2 and MEST DMRs were not significant between the groups studied. The H19 and IG-GTL2 DMR are paternally methylated DMRs (Kerjean et al. 2000, Geuns et al. 2007); however, our results suggest the H19 DMR is more prone to the loss of methylation compared with the IG-GTL2 DMR. The propensity of the H19 DMR to disturbances of DNA methylation may be related to the molecular structure of the DMR or of the surrounding sequences. The H19 DMR is less repetitive compared with the IG-GTL2 DMR (Paulsen et al. 2001) and it has been suggested that DNA methylation at more repetitive regions is more strictly conserved compared with regions that are less repetitive in nature, such as the H19 DMR (Li et al. 2004).

Our observation of a low rate of abnormal methylation in NOA samples is consistent with the published data (Hartmann et al. 2006, Marques et al. 2010). Due to the small sample size of NOA samples analyzed, the nonsignificant difference in methylation at imprinted genes in NOA men needs to be confirmed in future studies. Marques et al. (2010) reported a significant difference in methylation in NOA and OA men at the H19 and MEST DMRs. However, amplification was limited to one reaction resulting in a lack of variability in the clones analyzed (Marques et al. 2010), which suggests that cells carrying the normal imprint may have been preferentially amplified misrepresenting the methylation status of different sperm cells. We performed an average of 4.4 amplification reactions and analyzed unique clones, most of which originated from different amplification reactions (Fig. 1). This approach may facilitate analysis of DNA methylation in limited quantities of biological material to ensure that the results are representative of different cells. It is currently unknown whether the aberrant DNA methylation observed is specific to imprinted genes or also affects other sequences, including repetitive sequences and non-imprinted genes. However, detailed analyses may be challenging since a limited quantity of sperm can be isolated from the small amount of testicular tissue biopsied that is available for research purposes. 


\section{Analysis of methylation in testicular sperm}

The two published studies on DNA methylation at imprinted genes in testicular sperm of men affected by azoospermia did not include proper controls (Hartmann et al. 2006, Marques et al. 2010). We intended to use testicular sperm isolated from previously fertile men undergoing vasectomy reversal as controls for testicular sperm of azoospermic men. However, we found hypomethylated clones at the H19 DMR and hypermethylated clones at the MEST DMR in the sperm of these men. More appropriate controls may have been testicular sperm obtained from men undergoing vasectomy before any changes in testicular environment may occur (Jones 2004, McVicar et al. 2005). However, it is uncertain whether ethical approval would be granted for the biopsy of testicular tissue in men undergoing a vasectomy. In such cases, testicular biopsy would be a clinically unnecessary procedure and the risks involved with the procedure may not be justifiable for research purposes.

We compared DNA methylation at the DMRs of imprinted genes between testicular sperm and ejaculate sperm of unobstructed proven fertile men. DNA methylation at imprinted genes is established before cells enter meiosis and is maintained throughout development (Kerjean et al. 2000). Further changes in DNA methylation in the sperm occur during epididymal transit; however, these changes are limited to nonimprinted genes (Ariel et al. 1994). While methylation at non-imprinted genes can differ between testicular and ejaculate sperm, the methylation at imprinted genes has been shown not to differ between the two cell type populations in fertile unobstructed individuals (Kerjean et al. 2000, Hartmann et al. 2006). Therefore, the observed differences in methylation at the H19 DMR found between azoospermic men, men undergoing vasectomy reversal, and men of proven fertility are unlikely to be confounded by the sperm source. The observed changes in DNA methylation were associated with obstruction as significant changes in methylation were found in $\mathrm{OA}$ men and in men undergoing vasectomy reversal. Previous studies have reported the presence of imprinting abnormalities or hypomethylation at imprinted genes in the sperm of unobstructed normozoospermic men used as controls (Kobayashi et al. 2007, Poplinski et al. 2009, Boissonnas et al. 2010). However, abnormal methylation in these men may be related to subfertility (Ludwig et al. 2005), as these men were not of proven fertility. Sperm DNA methylation at imprinted genes appears well conserved in unobstructed men of proven fertility status, as suggested by our data and those of Hammoud et al. (2010).

Our groups were not age matched; however, no agerelated changes in DNA methylation at the DMRs of imprinted genes have been reported in germ cells, either in spermatozoa (Flanagan et al. 2006) or in oocytes
(Lopes et al. 2009). Therefore, the increased age of the vasectomy reversal men compared with the proven fertile men is unlikely to be associated with the observed changes in DNA methylation at the H19 DMR. A significant age difference was also observed between the two groups of men affected by obstruction, both of which showed decreased DNA methylation at the H19 DMR. This result suggests that the observed changes in DNA methylation at imprinted genes were not associated with age. Although our analysis suggests an association between obstruction and abnormal methylation at imprinted genes, the findings should be confirmed in a larger trial. Specifically, the difference in methylation at the H19 DMR between men undergoing vasectomy reversal and proven fertile men was statistically significant for comparisons of two independent groups but the significance was lost after correction for multiple group testing.

\section{Etiology of abnormal DNA methylation in sperm}

Loss of methylation at imprinted genes in the sperm has been associated with mutations in Dnmt3a and Dnmt3l (Kaneda et al. 2004, Yaman \& Grandjean 2006); however, mutations in these genes could not be identified in infertile men showing aberrant imprinting (Kobayashi et al. 2009). Gene mutations in other enzymes involved in methylation are possible, including DNMT1 (Li et al. 1992) and MTHFR (Kelly et al. 2005). Furthermore, exposure to endocrine disruptors has been associated with aberrant sperm DNA methylation at imprinted and non-imprinted genes (Anway et al. 2005, Stouder \& Paoloni-Giacobino 2010). It is also possible that factors limited to the testicular environment may be responsible for abnormal methylation in patients with obstruction.

In our data set, aberrant imprinting was primarily observed in the testicular sperm of men affected by obstruction, $\mathrm{OA}$, and in those post vasectomy. The testicular environment may change as a result of obstruction in vasectomized and OA men, and affect spermatogenesis (Jones 2004, McVicar et al. 2005). These results suggest that aberrant DNA methylation at imprinted genes may not only be associated with impaired spermatogenesis, as previously reported (Kobayashi et al. 2007, Marques et al. 2008, Poplinski et al. 2009, Boissonnas et al. 2010), but perhaps with changes in testicular environment that occur as a result of obstruction. Reduction of spermatogenesis may be associated with testicular tissue destruction related to an increase in reactive oxygen species (ROS; Aydos et al. 1998) associated with DNA damage, including DNA strand breaks, as well as the generation of DNA base adducts (Franco et al. 2008). DNA base adducts have been shown to impede DNA methylation by interfering with proper function of DNMTs, leading to DNA hypomethylation (Tan \& Li 1990, Hepburn et al. 1991, 
Weitzman et al. 1994, Turk et al. 1995). However, it is currently unknown whether DNA methylation at imprinted genes can also be affected by ROS-induced DNA damage. Future studies should evaluate the mechanisms that lead to changes in DNA methylation.

We found aberrant imprinting primarily in azoospermic men affected by $\mathrm{OA}$ and in vasectomy reversal cases. The OA pathology is similar to that of vasectomy reversal cases in that both types of samples came from men with normal spermatogenesis, where the sperm cannot reach the ejaculate due to obstruction. Our results suggest that an altered testicular environment may disrupt DNA methylation at imprinted genes. Therefore, aberrant imprinting may not only be related to spermatogenesis failure, as previously shown, but may also be disrupted by environmental factors. Furthermore, our results also show that DNA methylation at the $\mathrm{H} 19$ DMR is particularly prone to methylation abnormalities in vasectomy reversal and azoospermic patients.

\section{Materials and methods}

\section{Sample collection}

Testicular biopsies were obtained from 17 men undergoing vasectomy reversal (VR01-VR17), who were previously fertile having had a child prior to undergoing vasectomy. Eighteen testicular biopsies were obtained from infertile men presenting with azoospermia (AZO; TP01-18). Azoospermic men were subdivided into two subgroups: OA (OA; TP01-10) or NOA (NOA; TP11-15). Patients were assigned to the OA subgroup based on the diagnosis of normal spermatogenesis (TP03-TP05, TP07, TP10), the presence of CFTR gene mutations associated with obstruction due to the CBAVD (TP01, TP02, TP08; had $\Delta 508$ mutation), or both (TP06; CBAVD due to the 5T allele). Patient TP09 was assigned to the OA subgroup, based on the presence of epididymal head calcification. Patients were assigned to the NOA subgroup, based on the pathological evaluation of spermatogenesis failure due to hypospermatogenesis or partial maturation arrest. The pathology results were not available for three patients (TP16-TP18). We were unable to obtain testicular sperm from proven fertile unobstructed men, as biopsies from these healthy individuals may not be ethically justifiable for research purposes. DNA methylation at imprinted genes in the study groups was compared with that in ejaculate sperm obtained from proven fertile unobstructed men (C01-C09). These men had fathered a child within 2 years of sample donation and showed normal semen parameters according to WHO criteria ( $\geq 20$ million sperm $/ \mathrm{ml}, \geq 50 \%$ motile sperm, and $\geq 30 \%$ normal sperm morphology) (WHO 2010). The individuals included in this study did not have $\mathrm{Y}$ chromosome microdeletions and had a normal male karyotype. They also did not have varicocele or a history of infection or trauma. Ethical approval was obtained from the University of British Columbia Ethics Committee before initiating this study and all patients gave informed, written consent.

\section{Patient information}

Age was available for 13 of the 17 vasectomy reversal cases and 12 of the 18 azoospermic patients ( 9 of the $10 \mathrm{OA}$ and 3 of the $5 \mathrm{NOA}$ ). The mean age \pm s.D. (range) for the vasectomy reversal patients was $46.2 \pm 4.0(41-53)$ years. The mean age \pm s.D. (range) for the azoospermic patients was $37.8 \pm 6.9(28-51)$ years: $37.0 \pm 5.3(28-46)$ years for the OA group and 40.3 $\pm 11.6(28-51)$ years for the NOA group. The mean age \pm s.D. (range) for the proven fertile men was $34.1 \pm 2.4$ (30-38) years.

\section{Preparation of DNA}

Ejaculate sperm were isolated by standard swim-up. Prior to digestion, purity of the sample was evaluated under bright-field microscopy. DNA was digested according to Doerksen et al. (2000) and extracted using salt extraction. Between 200 and 350 testicular sperm were isolated by micromanipulation using an inverted microscope (Nikon, Tokyo, Japan) equipped with Hoffman modulating optics, a thermal stage and micromanipulators (Narishige, Tokyo, Japan).

The testicular sperm were deposited into a clean droplet of modified human tubule fluid (Irvine Scientific, Santa Ana, CA, USA). Sperm were micromanipulated using custommade micropipettes. Isolated clean sperm were transferred to a thin-walled $0.7 \mathrm{ml}$ microfuge tube (Sarstedt Ltd, Montreal, QC, Canada) and lyzed in $20 \mu \mathrm{l}$ of the alkaline and neutralization buffers, according to Manning et al. (2001). Experimental procedures were carried out on sperm cell populations devoid of other contaminating cells: ejaculate and testicular sperm.

\section{Sodium bisulphite modification}

Bisulphite modification is a chemical treatment of DNA that deaminates non-methylated cytosines into uracils, while methylated cytosines remain unchanged. Post-amplification of the original status of DNA methylation at cytosine residues can be differentiated: methylated cytosines remain as cytosines, while non-methylated cytosines are read as thymidines. Bisulphite modification was either performed on extracted DNA or on lyzed sperm cells split into two aliquots of $20 \mu \mathrm{l}$, using the EZ DNA Methylation-Gold Kit (Zymo Research, Orange, CA, USA), according to the manufacturer's instructions but with a shortened 2-h incubation period to limit degradation of the small amount of DNA used. Samples with a conversion rate of $95 \%$ or above were included in the analysis: modification performed either on ejaculate and testicular sperm provided an equally high conversion rate.

\section{Gene amplification and cloning}

DNA methylation was studied at two DMRs of imprinted genes methylated in the sperm, H19 and IG-GTL2, and at a DMR that is non-methylated in the sperm, MEST. Previously published primer sequences were used for the amplification of the selected sequences (Kerjean et al. 2000, Geuns et al. 2007). Amplification was carried out as outlined by Kerjean et al. (2000). Seventeen CpG loci were analyzed within the H19 
DMR. The known C/T single-nucleotide polymorphism (SNP) (SNP \#1073516) at CpG 7 was excluded from methylation analysis. Fifteen CpG loci were amplified within the IG-GTL2 DMR, but only the last ten CpG loci were analyzed due to the generation of truncated product, and $21 \mathrm{CpG}$ loci were amplified and analyzed within the MEST DMR. PCR products of the correct size were cloned into the pGEM-T Easy Vector System (Promega) to allow for blue/white colony screening. Two to three white colonies were picked per plate so that around ten white colonies were picked for each gene.

\section{Analysis of sequencing data}

Samples were submitted for sequencing to the McGill University and Génome Québec Innovation Centre (Montreal, QC, Canada). Products were sequenced with the SP6 sequencing primer using the Applied Biosystems 3730xl technology (Applied Biosystems, Inc., Foster City, CA, USA).

Sequences were aligned using ClustalW2 (Larkin et al. 2007). DNA methylation within sequences was analyzed manually. Differences among sequences were used to determine the unique status of each clone analyzed and included different methylation patterns at CpG loci, single nucleotide changes within the sequences, and having originated from a different amplification reaction. Unique clones originate from different sperm cells and may be more representative of the methylation status of different sperm cells and the sample. Preferential amplification may occur when small quantities of starting material are used (Walsh et al. 1992, Findlay et al. 1995); therefore to prevent this from occurring, multiple amplification reactions were set up per gene for each sample and unique clones were analyzed.

\section{Data analysis}

To compare DNA methylation at imprinted genes between groups, the methylation level for each sample was calculated based on the number of methylated CpGs in proportion to the total number of CpGs analyzed at unique clones within each DMR analyzed. This analysis provided a percent methylation value. The methylation level has been previously used as a quantifiable measure of DNA methylation assessed by bisulphite sequencing and used to compare differences in sperm DNA methylation between groups (Kobayashi et al. 2007, 2009). Differences in the methylation level among groups were determined using the non-parametric Mann-Whitney $U$ test or the Kruskal-Wallis test with Dunn's post hoc test for comparisons among more than two groups, as described by Poplinski et al. (2009). One-tailed $P$ values $<0.05$ were considered significant. One-tailed $P$ values were used as per our priori.

We determined the number of individuals with aberrant imprinting in each group. These men were defined as having at least one hypomethylated clone, at the H19 or IG-GTL2 DMRs, or hypermethylated clone at the MEST DMR. Hypomethylated clones were identified as having $<50 \%$ methylated CpGs at the H19 DMR and the IG-GTL2 DMR, while hypermethylated clones were identified as having more than $50 \%$ of methylated CpGs at the MEST DMR, as defined by Marques et al. (2010).
Differences in the number of individuals with abnormal methylation between groups were determined using Fisher's exact test. One-tailed $P$ values $<0.05$ were considered significant. One-tailed $P$ values were used as per our priori.

The unpaired two-tailed $t$-test was used to determine significant age differences between groups. Statistical analysis was performed using GraphPad Prism (version 5.02) (GraphPad Software, San Diego, CA, USA).

\section{Declaration of interest}

The authors declare no conflict of interest that could be perceived as prejudicing the impartiality of the research reported.

\section{Funding}

This work was supported by the Canadian Institute of Health Research (CIHR) (BMH-86005 and OBM-101387 to S Ma).

\section{Acknowledgements}

We gratefully acknowledge the patients who participated in this study. AM is a recipient of a graduate studentship award from the Interdisciplinary Women's Reproductive Health Research and the Canada Graduate Scholarship from the Natural Sciences and Engineering Research Council of Canada.

\section{References}

Anway MD, Cupp AS, Uzumcu M \& Skinner MK 2005 Epigenetic transgenerational actions of endocrine disruptors and male fertility. Science 308 1466-1469. (doi:10.1126/science.1108190)

Ariel M, Cedar H \& McCarrey J 1994 Developmental changes in methylation of spermatogenesis-specific genes include reprogramming in the epididymis. Nature Genetics 7 59-63. (doi:10.1038/ng0594-59)

Aydos K, Kupeli B, Soygur T, Unsal A, Erden E, Tulunay O \& Kupeli S 1998 Analysis of the relationship between histologic alterations and the generation of reactive oxygen species in vasectomized rat testes. Urology 51 510-515. (doi:10.1016/S0090-4295(97)00718-8)

Boissonnas CC, Abdalaoui HE, Haelewyn V, Fauque P, Dupont JM, Gut I, Vaiman D, Jouannet P, Tost J \& Jammes H 2010 Specific epigenetic alterations of IGF2-H19 locus in spermatozoa from infertile men. European Journal of Human Genetics 18 73-80. (doi:10.1038/ejhg. 2009.117)

DeChiara TM, Efstratiadis A \& Robertson EJ 1990 A growth-deficiency phenotype in heterozygous mice carrying an insulin-like growth factor II gene disrupted by targeting. Nature 345 78-80. (doi:10.1038/345078a0)

Doerksen T, Benoit G \& Trasler JM 2000 Deoxyribonucleic acid hypomethylation of male germ cells by mitotic and meiotic exposure to 5-azacytidine is associated with altered testicular histology. Endocrinology 141 3235-3244. (doi:10.1210/en.141.9.3235)

Findlay I, Ray P, Quirke P, Rutherford A \& Lilford R 1995 Allelic drop-out and preferential amplification in single cells and human blastomeres: implications for preimplantation diagnosis of sex and cystic fibrosis. Human Reproduction 10 209-218. (doi:10.1093/molehr/1.4.209)

Flanagan JM, Popendikyte V, Pozdniakovaite N, Sobolev M, Assadzadeh A, Schumacher A, Zangeneh M, Lau L, Virtanen C, Wang SC et al. 2006 Intra- and interindividual epigenetic variation in human germ cells. American Journal of Human Genetics 79 67-84. (doi:10.1086/504729)

Franco R, Schoneveld O, Georgakilas AG \& Panayiotidis MI 2008 Oxidative stress, DNA methylation and carcinogenesis. Cancer Letters 266 6-11. (doi:10.1016/j.canlet.2008.02.026) 
Georgiades P, Watkins M, Surani MA \& Ferguson-Smith AC 2000 Parental origin-specific developmental defects in mice with uniparental disomy for chromosome 12. Development 127 4719-4728.

Georgiades P, Watkins M, Burton GJ \& Ferguson-Smith AC 2001 Roles for genomic imprinting and the zygotic genome in placental development. PNAS 98 4522-4527. (doi:10.1073/pnas.081540898)

Geuns E, De Temmerman N, Hilven P, Van Steirteghem A, Liebaers I \& De Rycke M 2007 Methylation analysis of the intergenic differentially methylated region of DLK1-GTL2 in human. European Journal of Human Genetics 15 352-361. (doi:10.1038/sj.ejhg.5201759)

Hammoud SS, Purwar J, Pflueger C, Cairns BR \& Carrell DT 2010 Alterations in sperm DNA methylation patterns at imprinted loci in two classes of infertility. Fertility and Sterility 94 1728-1733. (doi:10.1016/j. fertnstert.2009.09.010)

Hartmann S, Bergmann M, Bohle RM, Weidner W \& Steger K 2006 Genetic imprinting during impaired spermatogenesis. Molecular Human Reproduction 12 407-411. (doi:10.1093/molehr/gal040)

Hepburn PA, Margison GP \& Tisdale MJ 1991 Enzymatic methylation of cytosine in DNA is prevented by adjacent $O-6$-methylguanine residues. Journal of Biological Chemistry 266 7985-7987.

Jarow JP, Espeland MA \& Lipshultz LI 1989 Evaluation of the azoospermic patient. Journal of Urology 142 62-65.

Jones R 2004 Sperm survival versus degradation in the mammalian epididymis: a hypothesis. Biology of Reproduction 71 1405-1411. (doi:10.1095/biolreprod.104.031252)

Kagami M, Nagai T, Fukami M, Yamazawa K \& Ogata T 2007 Silver-Russell syndrome in a girl born after in vitro fertilization: partial hypermethylation at the differentially methylated region of PEG1/MEST. Journal of Assisted Reproduction and Genetics 24 131-136. (doi:10.1007/s10815006-9096-3)

Kanber D, Buiting K, Zeschnigk M, Ludwig M \& Horsthemke B 2009 Low frequency of imprinting defects in ICSI children born small for gestational age. European Journal of Human Genetics 17 22-29. (doi:10.1038/ejhg. 2008.177)

Kaneda M, Okano M, Hata K, Sado T, Tsujimoto N, Li E \& Sasaki H 2004 Essential role for de novo DNA methyltransferase Dnmt3a in paternal and maternal imprinting. Nature 429 900-903. (doi:10.1038/ nature02633)

Kaneko-Ishino T, Kuroiwa Y, Miyoshi N, Kohda T, Suzuki R, Yokoyama M, Viville S, Barton SC, Ishino F \& Surani MA 1995 Peg1/Mest imprinted gene on chromosome 6 identified by cDNA subtraction hybridization. Nature Genetics 11 52-59. (doi:10.1038/ng0995-52)

Kelly TL, Neaga OR, Schwahn BC, Rozen R \& Trasler JM 2005 Infertility in 5,10-methylenetetrahydrofolate reductase (MTHFR)-deficient male mice is partially alleviated by lifetime dietary betaine supplementation. Biology of Reproduction 72 667-677. (doi:10.1095/biolreprod.104.035238)

Kerjean A, Dupont JM, Vasseur C, Le Tessier D, Cuisset L, Pàldi A, Jouannet P \& Jeanpierre M 2000 Establishment of the paternal methylation imprint of the human H19 and MEST/PEG1 genes during spermatogenesis. Human Molecular Genetics 9 2183-2187. (doi:10. 1093/hmg/9.14.2183)

Kobayashi H, Sato A, Otsu E, Hiura H, Tomatsu C, Utsunomiya T, Sasaki H, Yaegashi N \& Arima T 2007 Aberrant DNA methylation of imprinted loci in sperm from oligospermic patients. Human Molecular Genetics 16 2542-2551. (doi:10.1093/hmg/ddm187)

Kobayashi H, Hiura H, John RM, Sato A, Osu E, Kobayashi N, Suzuki R, Suzuki F, Hayashi C, Utsunomiya T et al. 2009 DNA methylation errors at imprinted loci after assisted conception originate in the parental sperm. European Journal of Human Genetics 17 1582-1591. (doi:10.1038/ejhg. 2009.68)

Larkin MA, Blackshields G, Brown NP, Chenna R, McGettigan PA, McWilliam H, Valentin F, Wallace IM, Wilm A, Lopez R et al. 2007 Clustal W and Clustal X version 2.0. Bioinformatics 23 2947-2948. (doi:10.1093/bioinformatics/btm404)

Li E, Bestor TH \& Jaenisch R 1992 Targeted mutation of the DNA methyltransferase gene results in embryonic lethality. Cell 69 915-926. (doi:10.1016/0092-8674(92)90611-F)

Li JY, Lees-Murdock DJ, Xu GL \& Walsh CP 2004 Timing of establishment of paternal methylation imprints in the mouse. Genomics 84 952-960. (doi:10.1016/j.ygeno.2004.08.012)
Lopes FL, Fortier AL, Darricarrère N, Chan D, Arnold DR \& Trasler JM 2009 Reproductive and epigenetic outcomes associated with aging mouse oocytes. Human Molecular Genetics 18 2032-2044. (doi:10. 1093/hmg/ddp127)

Lucifero D, Mertineit C, Clarke HJ, Bestor TH \& Trasler JM 2002 Methylation dynamics of imprinted genes in mouse germ cells. Genomics 79 530-538. (doi:10.1006/geno.2002.6732)

Ludwig M, Katalinic A, Gross S, Sutcliffe A, Varon R \& Horsthemke B 2005 Increased prevalence of imprinting defects in patients with Angelman syndrome born to subfertile couples. Journal of Medical Genetics 42 289-291. (doi:10.1136/jmg.2004.026930)

Manning M, Lissens W, Weidner W \& Liebaers I 2001 DNA methylation analysis in immature testicular sperm cells at different developmental stages. Urologia Internationalis 67 151-155. (doi:10.1159/000050972)

Marques CJ, Costa P, Vaz B, Carvalho F, Fernandes S, Barros A \& Sousa M 2008 Abnormal methylation of imprinted genes in human sperm is associated with oligozoospermia. Molecular Human Reproduction 14 67-74. (doi:10.1093/molehr/gam093)

Marques CJ, Francisco T, Sousa S, Carvalho F, Barros A \& Sousa M 2010 Methylation defects of imprinted genes in human testicular spermatozoa. Fertility and Sterility 94 585-594. (doi:10.1016/j.fertnstert.2009.02.051)

McVicar CM, O'Neill DA, McClure N, Clements B, McCullough S \& Lewis SE 2005 Effects of vasectomy on spermatogenesis and fertility outcome after testicular sperm extraction combined with ICSI. Human Reproduction 20 2795-2800. (doi:10.1093/humrep/dei138)

Olek A \& Walter J 1997 The pre-implantation ontogeny of the H19 methylation imprint. Nature Genetics 17 275-276. (doi:10.1038/ng1197-275)

Paulsen M, Takada S, Youngson NA, Benchaib M, Charlier C, Segers K, Georges M \& Ferguson-Smith AC 2001 Comparative sequence analysis of the imprinted Dlk1-Gtl2 locus in three mammalian species reveals highly conserved genomic elements and refines comparison with the Igf2-H19 region. Genome Research 11 2085-2094. (doi:10.1101/gr.206901)

Poplinski A, Tüttelmann F, Kanber D, Horsthemke B \& Gromoll J 2009 Idiopathic male infertility is strongly associated with aberrant methylation of MEST and IGF2/H19 ICR1. International Journal of Andrology 32 1-8. (doi:10.1111/j.1365-2605.2008.00924.x)

Raman R \& Narayan G 1995 5-Aza deoxyCytidine-induced inhibition of differentiation of spermatogonia into spermatocytes in the mouse. Molecular Reproduction and Development 42 284-290. (doi:10.1002/ mrd.1080420304)

Stouder C \& Paoloni-Giacobino A 2010 Transgenerational effects of the endocrine disruptor vinclozolin on the methylation pattern of imprinted genes in the mouse sperm. Reproduction 139 373-379. (doi:10.1530/ REP-09-0340)

Tan NW \& Li BF 1990 Interaction of oligonucleotides containing 6-O-methylguanine with human DNA (cytosine-5-)-methyltransferase. Biochemistry 29 9234-9240. (doi:10.1021/bi00491a018)

Turk PW, Laayoun A, Smith SS \& Weitzman SA 1995 DNA adduct 8-hydroxyl-2'-deoxyguanosine (8-hydroxyguanine) affects function of human DNA methyltransferase. Carcinogenesis 16 1253-1255. (doi:10. 1093/carcin/16.5.1253)

Walsh PS, Erlich HA \& Higuchi R 1992 Preferential PCR amplification of alleles: mechanisms and solutions. Genome Research 1 241-250. (doi:10.1101/gr.1.4.241)

Weitzman SA, Turk PW, Milkowski DH \& Kozlowski K 1994 Free radical adducts induce alterations in DNA cytosine methylation. PNAS 91 1261-1264. (doi:10.1073/pnas.91.4.1261)

World Health Organization (WHO) 2010 Sperm preparation techniques. In WHO Laboratory Manual for the Examination and Processing of Human Semen, 5 edn, pp 161-168. Geneva: World Health Organization.

Yaman R \& Grandjean V 2006 Timing of entry of meiosis depends on a mark generated by DNA methyltransferase3a in testis. Molecular Reproduction and Development 73 390-397. (doi:10.1002/mrd.20430)

Yoder JA, Walsh CP \& Bestor TH 1997 Cytosine methylation and the ecology of intragenomic parasites. Trends in Genetics 13 335-340. (doi:10.1016/S0168-9525(97)01181-5)

Received 12 January 2011

First decision 9 March 2011

Accepted 9 March 2011 